\title{
IdeAs
}

Idées d'Amériques

$6 \mid 2015$

Migrer dans les Amériques

\section{Les Boliviens aux États-Unis : tensions identitaires d'un groupe peu visible et hétérogène}

Bolivians in the United States, Identities in Tension within an Heterogeneous and Nearly Invisible Group

Los Bolivianos en Estados Unidos, tensiones identitarias de un grupo poco visible y heterogéneo

\section{Virginie Baby-Collin}

\section{(2) OpenEdition}

\section{Journals}

Édition électronique

URL : https://journals.openedition.org/ideas/1274

DOI : 10.4000/ideas. 1274

ISSN : 1950-5701

Éditeur

Institut des Amériques

Référence électronique

Virginie Baby-Collin, «Les Boliviens aux États-Unis : tensions identitaires d'un groupe peu visible et hétérogène », IdeAs [En ligne], 6| 2015, mis en ligne le 08 janvier 2016, consulté le 19 octobre 2022 URL : http://journals.openedition.org/ideas/1274 ; DOI : https://doi.org/10.4000/ideas.1274

Ce document a été généré automatiquement le 19 octobre 2022.

\section{c)}

Creative Commons - Attribution - Pas d'Utilisation Commerciale - Pas de Modification 4.0 International - CC BY-NC-ND 4.0

https://creativecommons.org/licenses/by-nc-nd/4.0/ 


\title{
Les Boliviens aux États-Unis : tensions identitaires d'un groupe peu visible et hétérogène
}

\author{
Bolivians in the United States, Identities in Tension within an Heterogeneous \\ and Nearly Invisible Group \\ Los Bolivianos en Estados Unidos, tensiones identitarias de un grupo poco visible \\ $y$ heterogéneo
}

Virginie Baby-Collin

\section{Introduction}

1 Les Hispaniques ou Latinos ${ }^{1}$ sont désormais la première minorité sur le sol états-unien ${ }^{2}$. Cette dénomination cache une très grande hétérogénéité de statuts et d'origines. On y trouve à la fois des citoyens états-uniens nés aux États-Unis (58\%) et des personnes nées à l'étranger ( $42 \%$ ), ayant acquis la nationalité états-unienne, des étrangers titulaires de visas multiples ou en situation d'irrégularité juridique ${ }^{3}$. Parmi ceux qui sont nés dans un pays latino-américain (21 millions), les plus nombreux viennent du Mexique (55\%), puis d'Amérique centrale et des Caraïbes, enfin d'Amérique du Sud (13\%).

2 Étant donné la diversité de cet ensemble hétérogène, existe-t-il un processus de construction d'une identification hispanique ou latina aux États-Unis, contribuant à identifier le groupe dans une société construite sur la différenciation ethno-raciale? Assignées et endossées, les identités, envisagées ici dans une perspective constructiviste, s'élaborent à la fois de l'extérieur, par le haut, via des processus d'hétéro-désignation émanant des acteurs sociaux, économiques et politiques dominants, et de l'intérieur, par le bas, via des processus d'auto-identification émanant des acteurs eux-mêmes. Celles-ci ne sont bien entendu pas fixes, ni essentielles, mais bien labiles et situationnelles, changeantes selon les situations, les interlocuteurs, les 
contraintes, les opportunités, les contextes spatiaux dans lesquelles elles prennent forme. En ce sens, la construction de la catégorie hispanique, et la façon dont elle peut être endossée par les acteurs, est bien spécifiquement située aux États-Unis, et se pose en des termes différents ailleurs. Mais elle se construit aussi différemment en Californie ou sur la côte Est, dans les lieux où le groupe est fortement visible ou plus discret : l'espace dans lequel ce processus prend place induit en effet des modes d'élaboration identitaire spécifiques.

C'est à cette question de la construction et de la mobilisation identitaire, des échelles et des espaces dans lesquels elle s'élabore et se met en scène, que s'intéresse ce propos, à partir de l'exemple de la migration bolivienne, dont la présence aux États-Unis a été peu étudiée jusqu'ici. Cet article répond ainsi à un double objectif. D'abord, il ambitionne de situer la migration bolivienne sur le sol américain, à la fois par rapport aux autres flux boliviens dans le monde, et par rapport aux autres flux latinoaméricains vers les États-Unis, afin d'en mettre en évidence les traits communs et les spécificités. Ensuite, il se propose de discuter les processus de construction identitaire à l'œuvre chez les migrants boliviens résidant sur le sol américain, à partir de leur principal espace d'installation, celui de la métropole de Washington, D.C.. Je m'attacherai à identifier comment s'exprime sur le sol américain la "bolivianité", entendue à la fois comme construction d'un sentiment d'appartenance à une communauté nationale imaginée (Anderson B., 1991), et comme mise en visibilité de cette appartenance commune, sur le sol états-unien, via des pratiques sociales spécifiques. Cette expression d'une appartenance à un groupe, via certaines pratiques, est supposée ici distincte de son observation en Bolivie ou dans d'autres espaces de la migration. Il s'agit enfin de mettre en évidence les autres échelles et supports d'identification des Boliviens en migration, tant à des niveaux scalaires inférieurs (échelles régionales, niveau des groupes sociaux ou ethniques) qu'à des niveaux supérieurs, notamment celui de l'identification au groupe hispanique.

Les sources mobilisées reposent sur l'exploitation de données statistiques, et sur l'analyse d'un matériau de terrain qualitatif recueilli auprès de migrants résidant aux États-Unis, dans les régions de Washington et de New York, entre 2010 et 2012. Une quarantaine d'entretiens auprès de migrants de différents profils, de représentants associatifs et institutionnels, ont été réalisés, couplés à des observations régulières d'activités réalisées par les migrants (dans le cadre associatif, sportif, festif en particulier), et à un suivi d'événements et activités partagés au sein des Boliviens aux États-Unis (groupes facebook, collectifs associatifs, manifestations diverses, moments de sociabilité).

\section{Les Boliviens aux États-Unis, hétérogénéité et concentration d'un petit groupe migrant}

\section{Les États-Unis, une destination secondaire dans le champ migratoire bolivien}

5 La Bolivie, l'un des pays aux indicateurs de développement les plus faibles d'Amérique du Sud, pointant au $108^{{ }^{e ̀ m e}}$ rang mondial pour son IDH (indicateur de développement humain), connaît une émigration (entre 6 et $7 \%$ de la population) supérieure à la moyenne régionale (autour de $5 \%$ ) qui assure des rentrées financières importantes vers 
le pays d'origine ${ }^{4}$, même si les données fluctuent d'une source à l'autre, étant donné les difficultés classiques de mesure relatives au phénomène migratoire (tableau 1).

Tableau 1 : Les principales destinations des migrants boliviens dans le monde

\begin{tabular}{|c|c|c|c|c|c|c|}
\hline Sources consultées & $\begin{array}{l}\text { Boliviens à } \\
\text { l'étranger }\end{array}$ & $\begin{array}{l}\text { Boliviens } \\
\text { en } \\
\text { Argentine }\end{array}$ & $\begin{array}{l}\text { Boliviens } \\
\text { en } \\
\text { Espagne }\end{array}$ & $\begin{array}{l}\text { Boliviens } \\
\text { aux États - } \\
\text { Unis }\end{array}$ & $\begin{array}{l}\text { Boliviens } \\
\text { au Brésil }\end{array}$ & $\begin{array}{l}\text { Boliviens } \\
\text { au Chili }\end{array}$ \\
\hline $\begin{array}{l}\text { PNUD et UN, } 2010 \text { (1) } \\
\text { Nombre de Boliviens } \\
\text { résidant en... } \\
\text { (\% du total) }\end{array}$ & 603000 & $\begin{array}{l}212786 \\
(35 \%)\end{array}$ & $\begin{array}{l}225707 \\
(37 \%)\end{array}$ & $\begin{array}{l}70784 \\
(12 \%)\end{array}$ & $\begin{array}{l}18287 \\
(3 \%)\end{array}$ & $\begin{array}{l}19092 \\
(3 \%)\end{array}$ \\
\hline $\begin{array}{lr}\text { Recensements des } \\
\text { pays d'installation } \\
\text { (date des } \\
\text { recensements selon } \\
\text { les pays) }\end{array}$ & Sans objet & $\begin{array}{l}345272 \\
(2010)(3)\end{array}$ & $\begin{array}{l}213872 \\
(2010)(4)\end{array}$ & $\begin{array}{l}77321 \\
(2010)(5)\end{array}$ & $\begin{array}{l}38828 \\
(2010)(6)\end{array}$ & $\begin{array}{l}25121 \\
(2012)(7)\end{array}$ \\
\hline $\begin{array}{l}\text { Recensement bolivien } \\
\text { de } 2012 \text { (2) } \\
\text { (émigration depuis } \\
2001) \\
\text { (\% du total des } \\
\text { émigrés) }\end{array}$ & $\begin{array}{l}487995 \\
(4,9 \% \text { de la } \\
\text { population })\end{array}$ & $\begin{array}{l}186512 \\
(38 \%)\end{array}$ & $\begin{array}{l}116520 \\
(24 \%)\end{array}$ & $\begin{array}{l}20491 \\
(4 \%)\end{array}$ & $\begin{array}{l}64340 \\
(13 \%)\end{array}$ & $\begin{array}{l}29021 \\
(6 \%)\end{array}$ \\
\hline
\end{tabular}

SOURCES :

(1) “UNITED NATIONS, DEPARTMENT OF ECONOMIC AND SOCIAL AFFAIRS (2013). TRENDS IN INTERNATIONAL MIgRANT STOCK: MIgRANTS BY DESTINATION AND ORIgIN (UNITED NATIONS DATABASE, POP/DB/MIG/STOCK/REV.2013)" : UNITED NATIONS DATABASE, POP/DB/MIG/STOCK/REV. 2013/ORIgIN

(2) Recensement bolivien : INE, 2012 (http://www.ine.gob.bo/)

(3) Recensement argentin : INDEC 2010 (www.indec.gov.ar)

(4) registre de population espagnol : INE 2010 (www.ine.es)

(5) recensement États-Unis : US Census 2010 (www.census.gov/2010census/)

(6) recensement brésilien : IBGE 2010 (www.ibge.gov.br)

(7) recensement chilien : INE 2012 (www.ine.cl)

6 Le champ migratoire bolivien se structure autour d'une polarité majeure ancienne, l'Argentine, destination depuis la fin du XIX ${ }^{e}$ siècle ; d'une migration régionale vers les autres pays voisins plus récente (Brésil, Chili) ; d'une émigration ayant connu une croissance très forte vers l'Europe au début des années 2000, principalement vers l'Espagne, secondairement vers l'Italie et d'autres pays de l'Europe occidentale ; enfin, autour d'une attractivité modérée des États-Unis, depuis une cinquantaine d'années. Il existe de très nombreux travaux sur la présence bolivienne en Argentine, où les Boliviens sont le second groupe migrant, derrière les Paraguayens (Caggiano S., 2005 ; Cortes G., 2008 ; Hinojosa A. et al., Le Gall J., 2011 ; Sassone S., 2002), ainsi qu'un certain nombre d'écrits relatifs à la présence bolivienne en Espagne (et secondairement en Italie), dont la croissance a été très rapide au début des années 2000, avant de connaître un mouvement de reflux lié à la crise espagnole de la fin de la décennie (Baby-Collin V., 
Cortes G., Miret M., 2009 ; Hinojosa A., 2009 ; Marzadro M., 2009 ; Parella Rubio S., 2003). En revanche, il n'existe que peu de recherches sur la présence bolivienne aux ÉtatsUnis, pour deux raisons principales. La première est que cette migration n'a jamais atteint des volumes suffisamment massifs pour susciter l'intérêt spécifique du monde de la recherche bolivianiste. Souvent mentionnée comme une destination secondaire, les travaux qui la considèrent l'ont plutôt observée au prisme des dynamiques transnationales et du lien entre migration et développement, envisageant les liens maintenus avec certaines communautés d'origine par les résidents aux États-Unis, et leur rôle dans le développement local en Bolivie (De la Torre Avila L., 2006 ; Jones R., De la Torre Avila L., 2011 ; Yarnall K., Price M., 2010). La seconde renvoie à la relative invisibilité du groupe au sein de la migration latino-américaine aux États-Unis. Si l'on écarte les innombrables travaux relatifs aux migrations mexicaines, centraméricaines et caribéennes, ceux consacrés aux groupes sud-américains aux États-Unis sont nettement moins nombreux. En ce qui concerne la présence bolivienne, soulignons ceux de Marie Price, sur les réseaux culturels et sportifs, ou l'entrepreneuriat ethnique dans la région de Washington (Price M., 2006 ; Price M., Chacko E., 2009 ; Price M., Whitworth C., 2004) et de Christopher Strunk (Strunk C., 2012, 2015), sur les pratiques de circulation des citoyennetés entre Washington et la région du Valle Alto de Cochabamba.

7 L'émigration bolivienne vers les États-Unis commence timidement après la Révolution bolivienne de 1952, puis après la réforme migratoire états-unienne de 1965, qui ouvre plus largement les portes à une immigration diversifiée. Les décennies 1960 et 1970, marquées par une très grande instabilité politique et une succession de régimes dictatoriaux, génèrent des flux modérés d'exilés politiques. Le profil de ces migrants est plutôt urbain, à fort capital social et culturel. Il est distinct de celui des migrants économiques dont le nombre augmente à partir des années 1980 : la crise économique de 1982 a particulièrement affecté la Bolivie, l'un des premiers pays latino-américains à adopter un plan d'ajustement structurel. L'émigration prend une importance nouvelle, dans le cadre régional (affirmation du pôle argentin, flux modérés vers le chili, le Brésil) et vers les États-Unis, avec des origines rurales, paysannes, mais aussi minières, et faiblement qualifiées. Il existe ainsi deux profils différenciés, tant dans les motivations du départ (migrations politiques / économiques), les origines géographiques (milieux urbains / plus ruraux et miniers), que le niveau socioéconomique (migrants qualifiés / peu ou pas qualifiés), dans les premières générations de migrants des années 1960 d'une part, 1980-90 d'autre part, même s'il existe aussi des porosités entre ces catégorisations.

8 Dans les années 2000, la Bolivie traverse plusieurs crises politiques et sociales guerres de l'eau, à Cochabamba puis à La Paz (2000), guerres du gaz (2003, puis 2005) (Poupeau F., 2008). Les conflits sociaux se doublent de tensions politiques entre Andes (à l'ouest) et basses terres (à l'est), alors que s'affirme le basculement économique du pays vers l'Oriente - les régions andines ayant centralisé richesses, populations et pouvoirs pendant des siècles (D'Andrea N., 2004 ; Franqueville A., 2000). Un bloc politique issu des départements de l'Oriente revendique une autonomie régionale accrue et s'oppose aux partis de gauche et aux représentants du monde indien / andin, le Movimiento al Socialismo (MAS) et son dirigeant Evo Morales Ayma, qui devient président de la République en 2006. Ces tensions favorisent de nouveaux départs depuis des régions jusqu'alors peu concernées par l'émigration (départements de Santa Cruz ou du 
Beni). La migration concerne de plus en plus des classes moyennes éduquées, dans lesquelles la composante féminine a une part croissante, plus marquée vers les destinations européennes (Espagne, Italie), où la demande de main-d'œuvre dans l'économie du care est forte. À destination des États-Unis, la difficulté croissante d'obtention des visas, depuis le 11 septembre 2001, ralentit les flux et constitue un filtre qui sélectionne les candidats à la migration, au profit des plus diplômés, de ceux qui disposent d'un certain capital économique, ou de réseaux familiaux permettant de justifier de procédures de regroupement familial. Le voyage et la traversée des frontières sans papiers constituent une épopée risquée et coûteuse qui décourage le plus grand nombre, comme le montre le faible nombre de départs vers les États-Unis dans les données du recensement bolivien de 2012 (tableau 1). La migration vers les États-Unis représente environ $10 \%$ de la migration bolivienne, mais seulement $4 \%$ de la migration récente (post 2001). Cette dernière est plus spécifiquement d'origine urbaine $^{5}$, et principalement issue des trois départements les plus peuplés du pays, où se concentrent les principales métropoles, La Paz, Cochabamba et Santa Cruz.

\section{La migration bolivienne aux États-Unis, un profil statistique majoritairement qualifié}

9 Aux États-Unis, les Boliviens représentent actuellement $3 \%$ des migrants sudaméricains (et $0,2 \%$ des migrants nés à l'étranger), soit environ 80000 personnes (dont la moitié sont devenus citoyens américains), et l'on recense 115000 personnes d'origine bolivienne dans le pays ${ }^{6}$, bien que les estimations consulaires évaluent à plus de 250000 le nombre de boliviens résidant uniquement dans la région de Washington (entretiens consulat bolivien de Washington, 2012). Les données de l'American Community Survey permettent de dresser le profil de cette population et de la mettre en perspective avec d'autres groupes significatifs (tableau 2).

Tableau 2 : Profils synthétiques des Boliviens par rapport à d'autres groupes migrants aux ÉtatsUnis

\begin{tabular}{|c|c|c|c|c|c|c|}
\hline & $\begin{array}{l}\text { Boliviens } \\
\text { nés à } \\
\text { l'étranger }\end{array}$ & 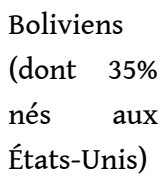 & $\begin{array}{l}\text { Total nés à } \\
\text { l'étranger }\end{array}$ & $\begin{array}{l}\text { Latino- } \\
\text { Américains } \\
\text { nés à } \\
\text { l'étranger }\end{array}$ & $\begin{array}{l}\text { Mexicains } \\
\text { nés à } \\
\text { l'étranger }\end{array}$ & $\begin{array}{l}\text { Sud- } \\
\text { Américains } \\
\text { nés à } \\
\text { l'étranger }\end{array}$ \\
\hline Âge médian & 43 & 32 & 41,5 & 39 & 37 & 42 \\
\hline$\%$ Femmes & 50 & 51 & 51 & 49 & 46 & 54 \\
\hline $\begin{array}{l}\text { Revenu médian } \\
\text { du foyer (US\$) }\end{array}$ & 58000 & 62000 & 49000 & 40000 & 37000 & 52000 \\
\hline $\begin{array}{l}\text { Taux de pauvreté } \\
\text { des familles (\%) }\end{array}$ & 9 & 6,5 & 17 & 23 & 28 & 12 \\
\hline
\end{tabular}




\begin{tabular}{|c|c|c|c|c|c|c|}
\hline $\begin{array}{l}\text { Population de } \\
\text { plus de } 25 \text { ans } \\
\text { ayant } \\
\text {-éducation } \\
\text { inférieure au } \\
\text { secondaire (\%) } \\
\text {-diplômes du } \\
\text { supérieur (\%) }\end{array}$ & $\begin{array}{l}13 \\
63\end{array}$ & $\begin{array}{l}12 \\
65\end{array}$ & $\begin{array}{l}31,5 \\
46.5\end{array}$ & $\begin{array}{l}47 \\
27\end{array}$ & $\begin{array}{l}60 \\
17\end{array}$ & $\begin{array}{l}17 \\
55\end{array}$ \\
\hline $\begin{array}{l}\text { Population de } \\
\text { plus de } 16 \text { ans } \\
\text { dans } \\
\text { population active } \\
\text { (\%) }\end{array}$ & 78,5 & 77 & 68 & 71 & 70 & 73 \\
\hline \multicolumn{7}{|c|}{ Activité (en \% de la population active occupée âgée de plus de 16 ans) } \\
\hline Agriculture & 0,3 & 0 & 3 & 4 & 7 & 1 \\
\hline $\begin{array}{l}\text { Construction, } \\
\text { industrie, } \\
\text { transport }\end{array}$ & 22 & 22 & 26 & 31 & 35 & 23 \\
\hline Commerce & 11 & 11 & 11 & 12 & 11 & 13 \\
\hline $\begin{array}{l}\text { Finance } \\
\text { professions } \\
\text { scientifiques }\end{array}$ & 17 & 19 & 18 & 15 & 14 & 19 \\
\hline Autres services & 49 & 50 & 41 & 38 & 33 & 44 \\
\hline
\end{tabular}

Source : élaboration propre à partir des données 2010 ACS 7 Y (American Community Survey 1 Year) et 2008-2012 ACS 5 Y estimates (American Community Survey 5 Years) - www.census.gov/programssurveys/acs/

La migration bolivienne, majoritairement familiale, présente un ratio hommes / femmes équilibré, qui la différencie des migrants mexicains, plus masculins, et sudaméricains, au profil plus féminisé. Les Boliviens se caractérisent par leur très haut niveau global de qualification et de diplômes, qui contraste avec celui des Mexicains en particulier ( $63 \%$ de Boliviens diplômés du supérieur, contre $17 \%$ des Mexicains), et par un taux d'activité très élevé. Une enquête montre que $31 \%$ des migrants originaires de la ville de La Paz à destination des États-Unis partent y faire leurs études (Ledo Garcia C., 2013). Les activités professionnelles sont majoritairement concentrées dans les services, et moins que les autres groupes dans la construction, le transport, l'industrie, ou l'agriculture (inexistante pour les Boliviens aux États-Unis). Ceci les différencie très fortement du profil bolivien en Argentine, moins qualifié, et plus inséré dans des activités peu ou pas qualifiées (agriculture, construction et commerce informel).

Ce profil médian plutôt qualifié et de classe moyenne des Boliviens aux États-Unis (taux de pauvreté trois fois inférieur à celui des Mexicains), assez proche de celui des autres originaires d'Amérique du Sud, est très différent du profil latino-américain majoritaire, 
lié à la domination mexicaine. Il diffère de celui des Boliviens des autres pôles du champ migratoire, en Argentine (où la migration est plus rurale et précaire) ou en Europe, où la migration est plutôt féminine, récente, moins stabilisée (Baby-Collin V., 2014b). Néanmoins, il convient de nuancer ce portrait, car les migrants sans papiers (souvent moins qualifiés et en situation plus précaire, bien qu'une observation fine mette en évidence une grande variété de configurations) sont en effet sous-représentés, quand le consulat de Bolivie à Washington estime leur proportion à la moitié du groupe présent sur le territoire.

\section{Concentration spatiale et métropolisation des Boliviens aux États- Unis}

12 La répartition spatiale des Boliviens aux États-Unis fait ressortir une logique classique de métropolisation migratoire, commune à l'ensemble des migrants, notamment à ceux originaires d'Amérique du Sud ; mais c'est aussi une géographie particulière qui témoigne d'une très forte concentration : près de la moitié des migrants boliviens (45\%) vivent dans l'aire métropolitaine de Washington, D.C., ce qui justifie l'étude de terrain réalisée dans cette région ; $13 \%$ vivent en Floride, $10 \%$ en Californie, $11 \%$ dans l'aire métropolitaine de New York. Cette géographie est très distincte de celle des Latino-Américains en général, fortement concentrés dans les États du Sud-Ouest - $40 \%$ des Latinos vivent en Californie et au Texas, puis dans la région de New York (15\%) et en Floride (14\%). Elle se distingue aussi de la migration sud-américaine plus concentrée dans les régions de New York (pour un tiers d'entre elle), de Floride, puis des États du Sud-Ouest, alors que la région de Washington, D.C. ne représente que $6 \%$ de la présence migrante sud-américaine. La consolidation d'une concentration bolivienne majeure dans la région métropolitaine de Washington renvoie à la structuration de filières migratoires, reposant sur des réseaux sociaux géographiquement situés, articulant espaces d'origine et d'arrivée selon des logiques relationnelles cumulatives, classiques des chaînes de la migration abondamment étudiées par la littérature (voir par exemple Faist T., 2000). 
Carte : Boliviens aux Etats Unis en 2010

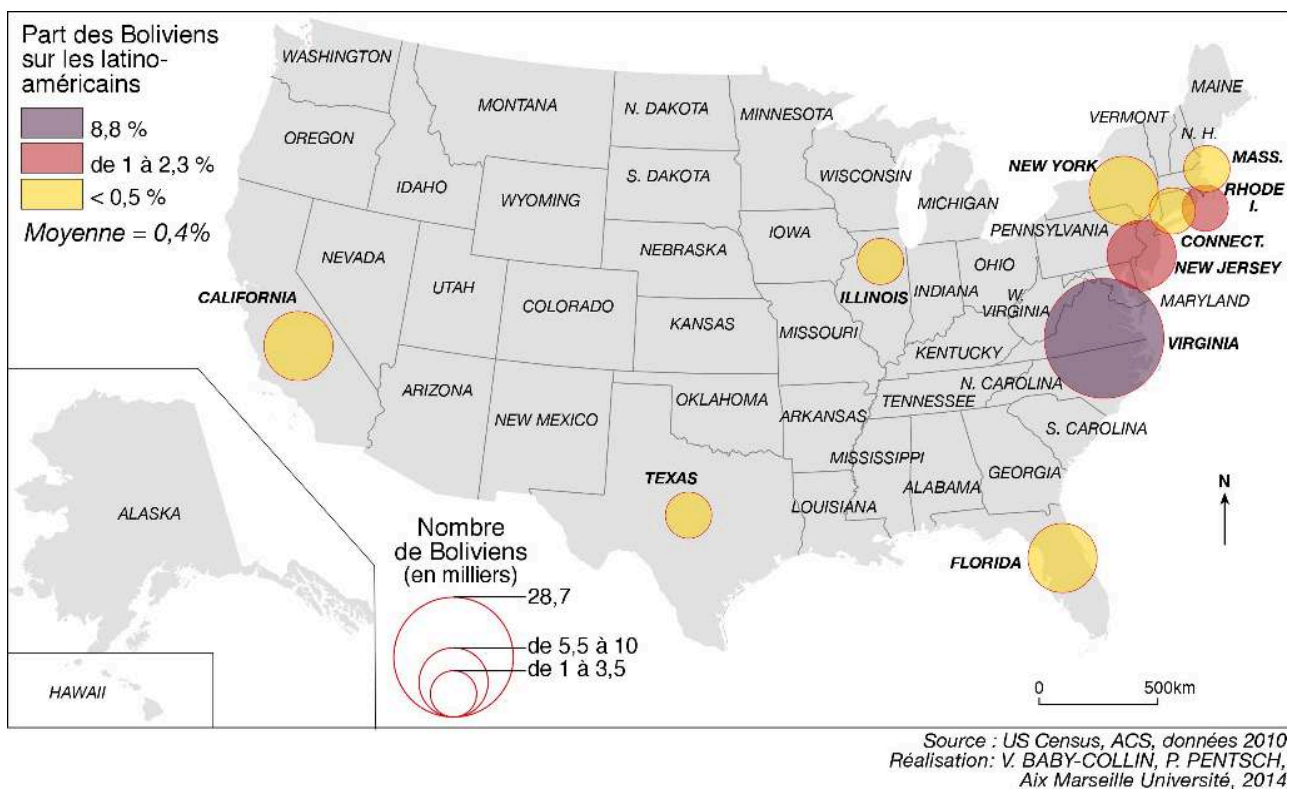

Source : US Census 2010 : www.census.gov/2010census/

Carte : Etrangers et Latino-américains aux Etats-Unis en 2010

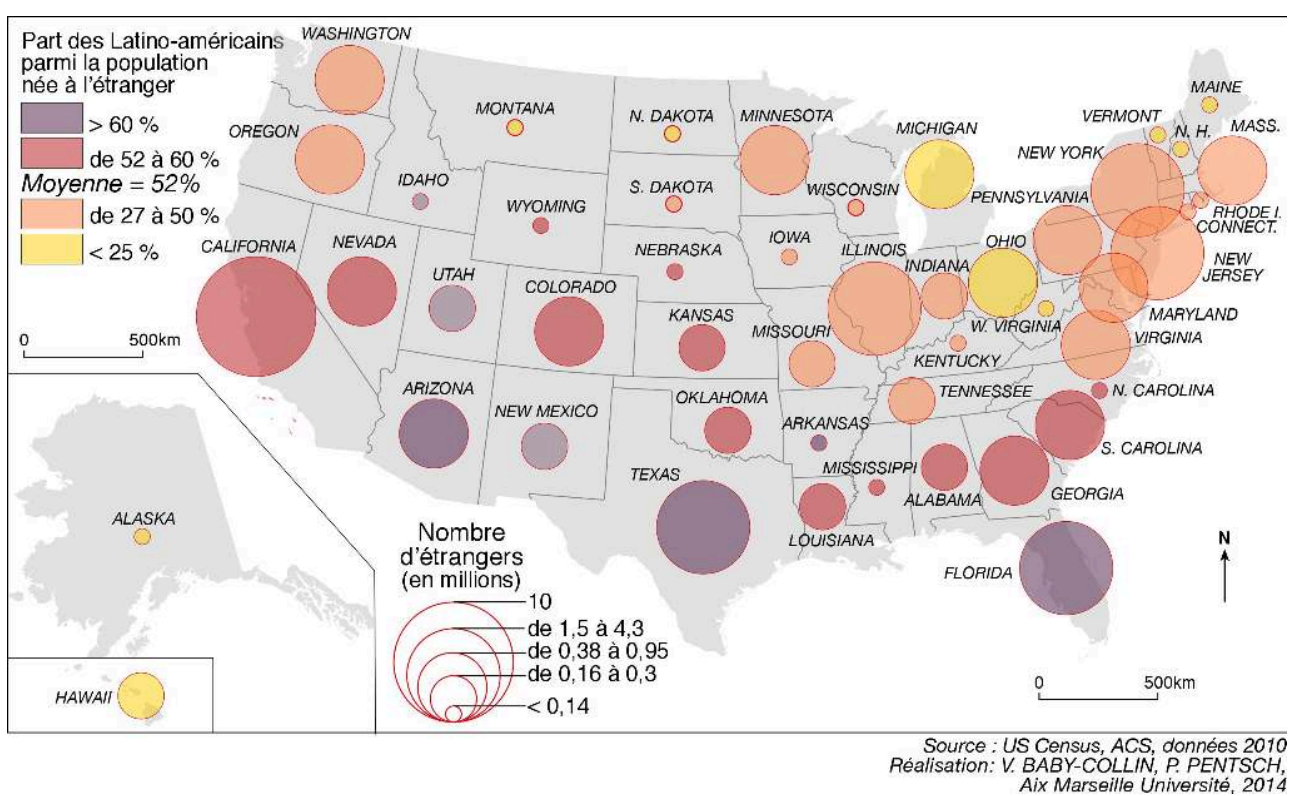

Source: 2010 ACS 1 Y (American Community Survey 1 Year)

La région métropolitaine de Washington, D.C. est un nouvel espace d'immigration : en 1970 , seulement $4 \%$ de sa population était étrangère ; en 2010 , avec $22 \%$ de population étrangère, elle se place au $7^{\text {ème }}$ rang pour l'accueil des étrangers en nombre (données US Census). La croissance de l'immigration (plus d'un million de nouveaux immigrants en 40 ans), sous tendue par un boom post-fordiste de l'économie des services, des technologies de l'information, du tourisme, de la construction, et des institutions fédérales (spécificité locale), a considérablement modifié le paysage métropolitain. Outre une proportion importante de migrants qualifiés, l'aire métropolitaine a accueilli 
un très grand nombre de réfugiés ; un tiers de la population migrante y est toutefois estimée sans papiers. La croissance des emplois qualifiés a généré des besoins dans les secteurs de la construction et des services non qualifiés, où les migrants sont particulièrement nombreux - plus d'un employé de la construction sur deux, dans la région, est étranger (Price M. et al., 2005).

Une spécificité de Washington est la très grande diversité des origines des étrangers, sans qu'aucune ne domine largement, au contraire d'autres grandes aires métropolitaines (Cubains à Miami, Mexicains à Los Angeles...) : le premier groupe est celui des Salvadoriens, mais ils ne représentent que $14 \%$ du total des étrangers. Parmi les Latino-Américains - les plus nombreux $(40 \%)$ - les Sud-Américains y ont précédé les Centraméricains et les Mexicains (croissance récente). On trouve aussi des nationalités asiatiques ( 5 des 6 premiers groupes en nombre) et africaines (Washington est la première ville éthiopienne aux États-Unis).

Contrairement aux portes d'entrée traditionnelles (New York, Los Angeles, Chicago par exemple), où les migrants ont commencé par investir des quartiers centraux, le caractère récent de la migration a ici favorisé une installation rapide des étrangers directement dans les banlieues. Il y a à la fois très peu d'enclaves ethniques de forte concentration d'étrangers issus d'un seul groupe, et beaucoup de quartiers caractérisés par l'hyperdiversité de leurs populations, bien que les degrés de dispersion varient selon les nationalités.

Carte 3 : Boliviens et Latino-américains dans l'aire métropolitaine de Washington en $2012^{7}$

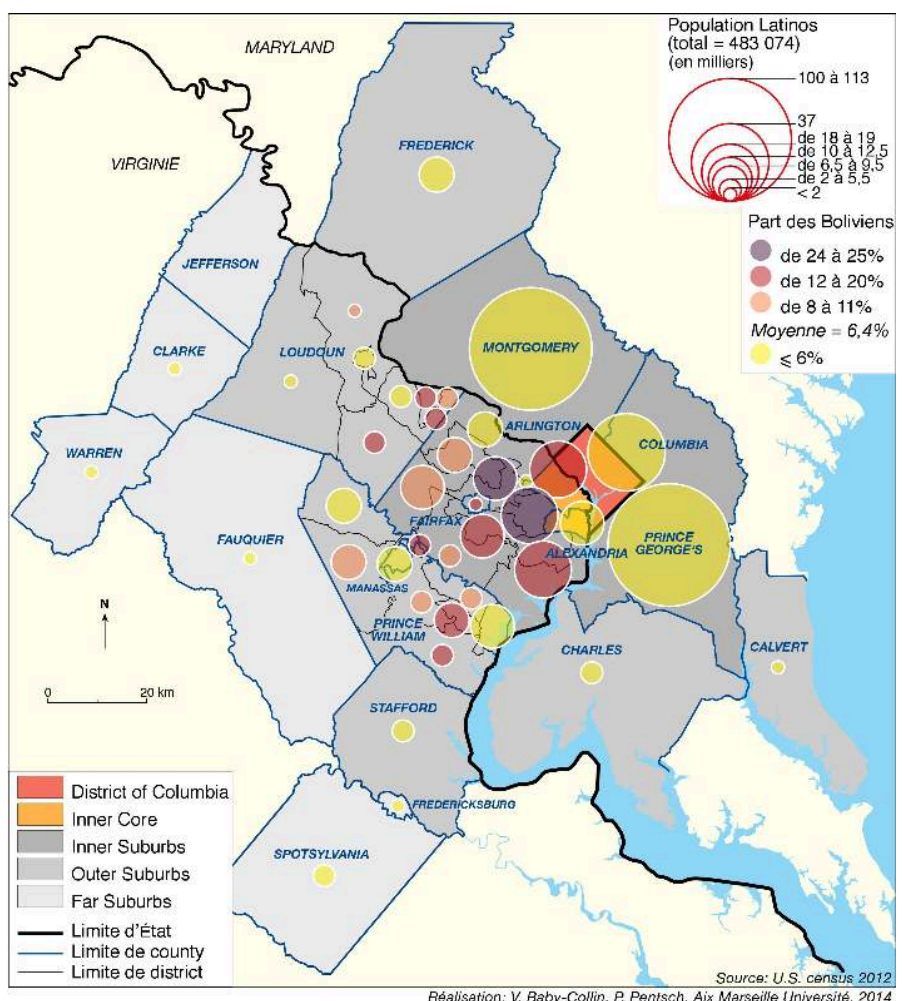

Source : 2008-2012 ACS 5 Y estimates (American Community Survey 5 Years) - www.census.gov/ programs-surveys/acs/

Les espaces de résidence des Boliviens sont assez fortement concentrés à l'ouest du Potomac, dans les espaces du inner core (municipalité d'Arlington) et les premières 
couronnes de banlieue (inner suburbs), dans le comté de Fairfax. Dans certains districts, les Boliviens représentent jusqu'à $25 \%$ des Latino-Américains. À partir d'Arlington, considérée comme le cœur de la présence bolivienne (le comté est jumelé avec la ville de Cochabamba, et surnommé Arlingtonbamba), on observe un processus de déconcentration résidentielle vers la banlieue plus lointaine, l'outer suburb (comtés de Prince William, Loudoun, Manassas). Dans ces banlieues traditionnellement blanches, le partage de l'espace Blancs / Latinos peut devenir source de conflits. Le groupe présente ainsi à la fois des espaces de concentration marqués et des dynamiques centrifuges.

\section{Les expressions collectives de la "bolivianité" aux États-Unis}

En Bolivie, les différences sociales sont très marquées, on ne se parle pas, on se méprise les uns les autres. Ici, on vit mieux ensemble parce qu'on a vu autre chose, parce qu'on a subi la même discrimination, alors on se retrouve. (Julia, 51 ans, 30 ans de résidence aux États-Unis. Entretien Arlington, 2011)

Si l'on accepte l'idée que l'identité est un construit social situé, il importe de montrer quels sont les ressorts de son élaboration. Comme nous invite à le penser Julia, être bolivien ne signifie pas la même chose en Bolivie et aux États-Unis. Quelles sont donc les expressions collectives de cette "bolivianité", mouvante selon les lieux où elle s'incarne, mais aussi les temporalités dans lesquelles elle s'inscrit ? En migration, l'expérience de la confrontation à l'altérité, de la stigmatisation, correspond à une hétéro-désignation négative, à laquelle peut répondre, de façon réactive, une autoidentification soudée d'un groupe qui ne se vivait pas forcément comme tel dans son pays d'origine. Néanmoins, cette identification bénéficie aussi d'un effet de concentration dans les espaces de la migration, et elle répond à des motivations diverses : besoin de retrouver l'air du pays à une quête de racines, réponse à la discrimination, valorisation d'une image positive de certains pans de la société d'origine, stratégiquement mise en scène. Elle évolue aussi, dans l'espace comme dans le temps, au contact de l'altérité qui à la fois lui donne sens et la transforme.

\section{Effets de lieux. Concentration spatiale et productions identitaires}

La présence bolivienne est quasiment invisible dans l'espace public états-unien pour qui est extérieur au groupe. Elle est en effet numériquement faible et noyée dans une masse de migrants latino-américains souvent peu différenciés de l'extérieur. Néanmoins, la forte concentration spatiale des Boliviens en certains quartiers de la région de Washington, D.C. favorise le maintien de liens intra-boliviens en migration, activant une identification du groupe par confrontation à l'altérité. À cet égard, l'expérience de Julia, qui a vécu en Californie avant de s'installer à Washington, souligne explicitement l'effet de concentration sur le sentiment d'appartenance à la Bolivie de ses filles:

En Californie, mes filles étaient seulement une latina de plus. Elles se sentaient hispanas, ou latinas si tu veux. C'était comme ça aussi qu'elles devaient être. Ici c'est un grand changement. Ici, elles sont devenues boliviennes, et elles peuvent exister comme telles. En Californie, il n'y a pas de Boliviens, ou alors ils sont éparpillés [....] Ici, on trouve tout ce qu'on veut de Bolivie ! [...] La communauté est très importante, les liens sont plus forts, on existe comme des Boliviens. En 
Californie les Boliviens sont plus éduqués, ils sont venus pour faire des études, ou bien avec leurs diplômes. Ici c'est différent, tu as tout le spectre de l'échelle sociale, c'est plus varié. Il y a des paysans, des diplômés... (Julia, 51 ans, réside depuis 30 ans aux États-Unis. Entretien 2011)

Soulignons que la diversité sociale est ici considérée comme dépassée par une identification à la Bolivie, capable de souder un groupe au-delà de ses origines différenciées. Ce processus est notamment revendiqué par les secondes générations (nées aux États-Unis, donc américaines de nationalité), y compris de milieux sociaux très favorisés. C'est ce dont témoigne Jorge, issu de l'élite de La Paz, qui a quitté la Bolivie dans les années 1970, à l'âge de vingt ans, pour finir ses études aux États-Unis (entretien Arlington, 2011). Il y est devenu ingénieur chez IBM. Marié à une Américaine, très intégré dans un monde états-unien où il a connu une réussite professionnelle remarquable, il n'a pas, pendant des années, entretenu de liens à la Bolivie, ni exprimé une appartenance autre que très individuelle et privée à son pays d'origine, ne souffrant pas de l'expérience de la discrimination, dont son statut et ses qualifications professionnelles l'ont préservé. Jusqu'à ce que sa fille, à l'adolescence, le questionne sur ses origines. Jorge, qui connaît l'existence de nombreuses associations culturelles boliviennes dans la région de Washington, décide d'y amener sa fille. Il y découvre le folklore bolivien, y renoue avec la pratique de sa langue, y retrouve sa culture. Son engouement l'amène à devenir président du comité Pro Bolivia, l'une des principales associations boliviennes, qui organise chaque année le festival bolivien de Washington. Sa fille finit aujourd'hui ses études en Latin American Studies à l'université.

Cet exemple illustre le rôle que la seconde génération peut avoir dans la recréation de liens identitaires à l'origine, tant pour les jeunes que pour les parents, et le rôle de la culture comme élément particulièrement performant pour reconstruire cette identité. Il signifie aussi l'effet de concentration du groupe dans le processus de reconnexion à la Bolivie.

\section{Pratiques d'entre-soi ethnique et promotion d'une appartenance commune}

21 Si l'affichage de la "bolivianité" est relativement faible dans l'espace public ordinaire, des sociabilités et des réseaux ethniques tissent dans les métropoles d'installation une toile relationnelle qui s'appuie sur certains moments, lieux et pratiques, lors desquels l'appartenance à un même groupe s'exprime et se donne à voir. Il s'agit souvent de formes discrètes, non pérennes, et articulées à la sphère des loisirs. C'est en entrant dans ces réseaux que le groupe devient visible, notamment les fins de semaine, moments privilégiés pour l'activation de formes d'entre- soi ethnique. Les activités sportives et associatives en sont deux exemples.

22 La pratique du football, chez les hommes comme chez les femmes, est populaire en Bolivie comme dans les espaces de la migration. Les matchs sont prétextes à d'autres échanges: on vient y manger des spécialités culinaires boliviennes préparées par les uns et les autres, discuter des dernières nouvelles du pays, s'informer des possibilités d'emploi et de logement, pour les nouveaux venus, discuter des prochains événements de la communauté locale.

23 Ces réseaux de sociabilité réunis régulièrement sur les stades, devenus des centralités ethniques hebdomadaires, se retrouvent dans les associations culturelles. La richesse 
du folklore andin et l'importance de sa pratique, dont la mode et la popularité n'ont fait que croître en Bolivie depuis plusieurs décennies, ont généré dans les espaces de la migration des associations culturelles qui préparent tout au long de l'année des spectacles, mis en scène publiquement lors des festivités emblématiques du calendrier : fête des mères, des enfants, anniversaire de la Bolivie, Carnaval, fêtes votives... Plus d'une quarantaine d'associations boliviennes de ce type ont été recensées dans la mégalopole états-unienne en 2012. Les répétitions ont lieu le soir, ou les fins de semaine, sur les parkings des centres commerciaux de la suburb, dans des locaux municipaux. Ces associations, qui entretiennent, activent ou recréent un sentiment d'appartenance national, ont aussi pour ceux qui y envoient leurs enfants des fonctions plus prosaïques : elles permettent la pratique de la langue espagnole hors du cadre domestique, et éloignent les jeunes du spectre de la délinquance urbaine latina qui hante les parents. Pour les adultes, les activités culturelles entretiennent ou réactivent un lien au pays, dans des dynamiques où se mêlent à la fois la nostalgie, la promotion et la valorisation d'un élément de fierté nationale, transformé ou réinventé en migration. Car la popularité des festivités boliviennes, qui dépasse le cadre du groupe pour toucher un public (latino ou non) plus large, institue la Bolivie en "terre du folklore", et véhicule aux États-Unis une représentation pour une fois positive d'un pays généralement associé à des imaginaires très négatifs : pauvreté, misère, sousdéveloppement, narco-trafic, tensions politiques, indianité, ruralité, retard. La promotion culturelle constitue en ce sens un renversement du stigmate, et permet, pour des migrants souffrant souvent de discriminations, de trouver sur la scène publique un espace privilégié de reconnaissance.

La transformation de la célébration de la fête de la Vierge d'Urkupiña ${ }^{8}$ dans la région de Washington illustre ce processus de mise en scène de ce qui devient progressivement un emblème local de la "bolivianité". Maria et Rodrigo ont ramené à Washington une statuette de la Vierge d'Urkupiña ${ }^{9}$ en 1987, lors de leur premier voyage de retour au pays (entretiens Washington, 2012), et fondé le Comité Pro Virgen de Urkupiña en 1988. Le 15 août 1989, sept groupes folkloriques accompagnés de musiciens rassemblent deux à trois cents familles boliviennes de la région et donnent vie au premier Festival boliviano, avec la célébration d'une messe dans la cathédrale de Washington, D.C. Le festival, qui a fêté ses vingt-cinq ans en 2013, s'est laïcisé ; il est désormais fêté le weekend de Labor Day pour toucher un public plus important que pendant la période estivale. Il s'est déplacé de l'inner core (Arlington) vers la périphérie (Manassas, au SudOuest de l'agglomération), où réside un grand nombre de Boliviens. Devenu une véritable entreprise, regroupant 2000 danseurs de la région et d'ailleurs aux ÉtatsUnis, le festival rassemble désormais plus de 10000 personnes. Il s'est converti en une immense fête populaire et culturelle, ouvert à un public latino plus large. La folklorisation de la fête en migration, détachée de son espace d'origine, passe ainsi par une recomposition de ses modalités d'expression, une commercialisation, et l'incorporation de pratiques culturelles du pays d'installation : à la mode américaine, on célèbre le festival bolivien des enfants, on organise des galas, des pique-niques, des concours de Miss... L'expression collective de l'appartenance bolivienne se recompose et se transforme ainsi dans l'espace migratoire. 


\section{Entre tensions internes et construction d'un univers latino. Multiscalarité des références identitaires}

La célébration de cette appartenance fonctionne, on l'observe dans l'expérience de la fête, comme outil d'identification et de communion en migration. Elle ne résiste cependant pas toujours face à l'affirmation de revendications identitaires régionalisées. Elle est aussi concurrencée par l'émergence d'une identification hispanique plus englobante, à la fois fruit d'un processus progressif d'incorporation où émerge un cosmopolitisme hispanique, motivée par la construction d'un marché latino, et activée comme ressource dans une société où la latinité est aussi stratégique.

\section{Tensions infra-nationales : identifications régionales et racisme de classe}

Les tensions régionales boliviennes entre Andes et basses terres, mentionnées plus haut, s'exportent avec la migration. Dans la région de Washington, l'arrivée plus récente des migrants issus de l'Oriente a généré des espaces de vie et de mise en scène des Cruceños $^{10}$ distincts de ceux des Andins, ces derniers, présents depuis plusieurs décennies, étant numériquement dominants dans l'espace métropolitain. Les quartiers de résidence ou les espaces de sociabilité des uns et des autres sont souvent disjoints, comme l'explique Sebastian, 42 ans, journaliste originaire du Béni, qui réside aux ÉtatsUnis depuis six ans : il évite la zone d'Arlington, car "il y a trop de Boliviens de Cochabamba" ; ceux "de l'Oriente" résidant plutôt, comme lui, dans les secteurs Annandale et Alexandria, parce que "nous les Cruceños, nous aimons bien être ensemble" (entretien 2011). Par l'utilisation récurrente du "Nous les Cruceños" tout au long de l'entretien, il exprime une revendication identitaire forte qui renvoie en creux à une différenciation marquée face à "Eux les Andins". Choqué du manque de reconnaissance, aux États-Unis, de la diversité bolivienne, il a fondé une association qui valorise et diffuse les us et coutumes de l'oriente, qui organise notamment le Carnaval bolivien de Washington et une grande fête pour le "Jour de la tradition cruceña", distincte du festival boliviano dans lequel il ne se reconnaît guère. Cette initiative résonne dans d'autres espaces de connexion des Cambas aux États-Unis, via des manifestations spécifiques: Carnavals (le carnaval de l'Oriente se distingue des fêtes andines), élections des Miss (affichage spécifique des traditions de l'Oriente, région réputée pour accueillir les plus jolies filles du pays). Les regroupements politiques des migrants issus de l'Oriente sont aussi en général opposés au MAS, le parti d'Evo Morales, comme le montrent les enquêtes réalisées sous la direction de Jean-Michel Lafleur sur le vote bolivien à l'extérieur (Lafleur J-M., 2012).

Les résultats des élections de 2009, les premières dans lesquelles le vote à l'extérieur des citoyens Boliviens a été autorisé, mettent en évidence la large domination d'un vote d'opposition à Evo Morales aux États-Unis, confirmé lors des élections 2014, faisant de ce pays l'un des rares lieux où le vote d'opposition domine. Bien que les votants à l'extérieur soient très peu nombreux (4 700 votants aux États-Unis aux élections de 2014), il s'en dégage un certain profil, diplômé, installé de longue date, d'origine urbaine et de l'Oriente (Lafleur J-M., Von Arx E., 2012), opposé au premier président indien du pays, Evo Morales étant d'origine aymara. 
De fait, le mépris des populations indiennes ${ }^{11}$, notamment par les élites boliviennes, très ancré dans l'histoire nationale, rejoue dans les espaces de la migration, doublé d'inégalités d'ordre socio-économique. L'élite économique bolivienne installée dans la région de Washington réside dans les quartiers huppés du Maryland et des abords du fleuve Potomac. Elle a fondé une association culturelle boliviano-américaine, qui, comme son nom l'indique, revendique une double appartenance, dans des familles qui ont généralement la double nationalité. Elle promeut une vision artistique de la culture, très éloignée de celle que défend le Comité Pro Bolivia, loin de la dimension folklorique de la culture populaire, issue de traditions associées au monde indien et andin. Elle n'est pas représentée dans les fêtes du folklore andin, et l'on raconte que dans les années 1990, cette élite a boudé le dîner de gala organisé en l'honneur de la visite officielle de la femme de Victor Hugo Cárdenas ${ }^{12}$, invitée par les réserves indiennes aux États-Unis, organisé à Washington par les Boliviens (entretiens 2011).

Ces tensions d'ordre social et ethnique renvoient à des inscriptions territoriales et des pratiques différenciées, et signifient l'exportation dans les espaces de la migration de clivages profonds de la société bolivienne.

\section{De Boliviens à Latinos : assignation "faute de combattants", cosmopolitisation de la société, ou ressource stratégique?}

Dans l'espace public, l'affichage commercial à destination de la clientèle bolivienne est rare et dispersé, ce qui contraste avec d'autres espaces de la migration bolivienne ${ }^{13}$, ou d'autres centralités commerciales visibles aux États-Unis, les quartiers chinois étant un exemple emblématique (Kaplan D., Li W., 2006). En raison de la faiblesse numérique du groupe, l'affichage se dilue dans une scénographie latino, au potentiel commercial plus important : une stratégie marketing d'ouverture faute de "combattants nationaux".

31 L'émergence sur le sol américain d'une forme de "racialisation latina" (Davila A., 2008) est visible dans la chute constante, dans les recensements, des Hispaniques qui s'identifient comme "Blancs" (64\% en 1980, 54\% en 1990, 48\% en 2000), au profit de ceux qui s'identifient comme relevant de "some other race" (Hispanic). On assiste à l'émergence d'un tripartisme racial, où le Latino trouve une place entre le Blanc et le Noir. L'identification latina, réactive et instrumentale, se construit sur le socle de sa marginalisation sociale, et élabore en retour des formes d'appartenance collective, dans des quartiers latinos multiethniques, des groupes luttant pour une meilleure reconnaissance de leur place dans la société états-unienne.

Dans les quartiers de résidence, le partage d'espaces de vie communs (école, travail), où se côtoient des nationalités diverses, favorise des interactions qui témoignent d'une cosmopolitisation populaire, par le bas, des sociétés urbaines, non pas entendue comme une utopie sociétale ou un horizon, mais bien comme un univers concret de situations (Amin A., 2002 ; Vertovec S., 2007 ; Webner P., 1999). La latinité se construit via un processus d'incorporation progressive des groupes dans la société états-unienne. Ainsi en est-il des inventions culturelles : le festival latino de Washington, D.C., qui existe depuis le début des années 1970, s'est développé dans un quartier à la présence latinoaméricaine marquée. L'identité hispanique est activée sur la scène de la représentation politique, dans des dynamiques tant top down que bottom up, car l'électorat latino (la majorité des Hispaniques est citoyenne des États-Unis) est courtisé par les candidats. 
33 Se présenter sous la bannière hispanique est aussi souvent la seule façon de pouvoir accéder à des représentations politiques dans des espaces pluriethniques. À Arlington, l'élection de la première femme hispanique au School Board en 2008 constitue un événement. L'élue est une Bolivienne, fondatrice d'une association locale, Escuela Bolivia, rebaptisée en 2010 "Educación para nuestro futuro / Education for our future", afin de gommer une identification nationale devenue un obstacle à sa diffusion dans un cercle latino plus large. De fait, la campagne électorale de l'élue s'est construite autour d'un discours visant à regrouper un électorat latino, condition sine qua non de son élection.

\section{Conclusion : des processus identitaires à l'épreuve de la distance et des temporalités}

Petit groupe migrant, aux caractéristiques statistiquement proches de celles des autres originaires d'Amérique du Sud résidant aux États-Unis, les Boliviens en migration se distinguent des Mexicains et Centraméricains. Ils apparaissent très concentrés dans certaines régions métropolitaines, dont Washington, D.C. est le cœur, loin des centralités mexicaines du sud-ouest. La migration est plus familiale, urbaine et qualifiée, que la présence bolivienne en Argentine, plus hétérogène en raison de son ancienneté (plus d'un siècle), plus rurale, indienne, et peu qualifiée. Mais les profils boliviens aux États-Unis sont aussi hétérogènes, à la fois quechuas des zones rurales, accédant difficilement au processus de régularisation, élites urbaines qualifiées devenues citoyennes américaines, et classes moyennes plus récemment arrivées aux situations très variables, sur le plan économique et juridique, et aux origines géographiques élargies.

La "bolivianité", comme expression et mise en visibilité, par le biais de certaines pratiques sociales, d'un sentiment d'appartenance national, s'exprime aux États-Unis principalement dans la région métropolitaine de Washington, D.C., où l'effet de concentration joue sur la capacité d'expression du groupe. Ses logiques sont plurielles : à la fois réactives, contribuant à une identification face à une altérité dominante, dans des contextes de discrimination variables; de type nostalgique, associées à une quête des racines, y compris pour des migrants de seconde génération ; des logiques relatives à l'expression quotidienne d'un entre-soi ethnique maintenu par le fonctionnement de réseaux sociaux, pour communiquer avec les siens, ou comme sociabilités ressources ; des logiques promotionnelles enfin, liées à la valorisation, notamment sur la scène culturelle, d'une image nationale généralement plutôt dépréciée dans la société d'installation.

Cette mise en visibilité n'est cependant pas exempte de dominations et de conflits, révélant que l'idée d'une communauté migrante ne résiste pas à l'épreuve de certaines pratiques du quotidien. La communauté nationale est à la fois nostalgie, ressource, force de revendication, mais aussi un imaginaire lézardé par la violence des relations sociales qui traversent un groupe hétérogène.

La latinité, comme enveloppe plus large d'appartenance, est un support d'identification progressivement endossé par les migrants, d'abord parce que fortement assigné. Par leur faiblesse numérique, les Boliviens invisibles se diluent dans un groupe hispanique construit par les catégories censitaires, sociétales, promu par les entrepreneurs en 
quête de marchés et de clients nouveaux, et par les cadres politiques dans lesquels l'appartenance ethnique est une ressource et un instrument stratégique. Mais l'incorporation progressive des Boliviens au sein des Latinos des États-Unis témoigne aussi d'un processus de cosmopolitisation sociale par ses acteurs quotidiens.

En tension, mais en construction, les identifications relèvent ainsi à la fois d'assignations par le haut, d'ordre politique et économique, mais aussi d'élaborations par le bas, dans les quartiers multiculturels où s'élaborent des cosmopolitismes, dans des fêtes où l'identité se met en scène, dans l'espace politique où elle devient stratégique. En ce sens, la latinité aux États-Unis est aussi une porte ouverte, une ressource à saisir, pour élaborer une revendication, une appartenance citoyenne.

\section{BIBLIOGRAPHIE}

Amin, Ash, « Ethnicity and the multicultural city: living with diversity », Environment and Planning A, vol. $34, \mathrm{n}^{\circ} 6,2002$, p. 959-980.

Anderson, Benedict, Imagined Communities: Reflections on the Origin and Spread of Nationalism, Revised, Verso, 1991.

Baby-Collin, Virginie, « Les sans-papiers, un enjeu multiscalaire pour les politiques migratoires aux États-unis ", BAGF (Bulletin de l'Association des Géographes Français), n², 2014a, p. 196-209.

Baby-Collin, Virginie, Prendre place ici et là-bas. Géographie multisituée des migrations boliviennes (Argentine, États-Unis, Espagne). Volume 3, inédit., Habilitation à Diriger des Recherches, Nanterre, Université Paris Ouest Nanterre la Défense, 2014b.

Baby-Collin, Virginie, Cortes Geneviève, Miret Naïk, « Les migrants andins en Espagne, inscriptions spatiales et repérage de filières ", Mélanges de la Casa de Velázquez, vol. 39, n¹, 2009, p. $115-140$.

Caggiano, Sergio, Lo que no entra en el crisol : inmigración boliviana, comunicación intercultural y procesos identitarios, Prometeo Libros Editorial, 2005.

Cortes, Geneviève, Migrations, espaces et développement. Une lecture des systèmes de mobilités et des constructions territoriales en Amérique Latine, Habilitation à Diriger des Recherches, Poitiers, 2008.

D'Andrea, Nicolas, Effets de frontière, migrations et redistribution du peuplement en Bolivie : les dynamiques territoriales dans le département du Tarija, Thèse de doctorat en aménagement et urbanisme, Université Aix Marseille III, 2005.

Davila, Arlene, Latino Spin. Public Image and the Whitewashing of Race, New York, New York University Press, 2008.

De La Torre, Avila Leonardo, No Llores, prenda, pronto volveré. Migración, movilidad social, herida familiar y desarrollo, La Paz, PIEB, IFEA, 2006.

Faist, Thomas, The volume and dynamics of international migration and transnational social spaces, Oxford - New York, Clarendon Press ; Oxford University Press, 2000. 
Franqueville, André, La Bolivie, d'un pillage à l'autre, Paris-Toulouse, IRD-Presses universitaires du Mirail, 2000.

Hinojosa, Alfonso, Buscando la vida. Familias bolivianas transnacionales en España, La Paz, CLACSOPIEB, 2009.

Hinojosa, Alfonso (dir.), Idas y venidas, campesinos tarijeños en el norte argentino, La Paz, PIEB, 2000.

Jones, Richard, De La Torre Avila Leonardo, « Disminished Tradition of Return? Transnational Migration in Bolivia's Valle Alto ", Global networks, vol. 11, n², 2011, p. 180-202.

Kaplan, Daniel, Li Wei, Landscapes of the Ethnic Economy, Lanham, Rowman \& Littlefield, 2006.

Lafleur, Jean-Marie (dir.), Diáspora y voto en el exterior. La participación poítica de los emigrantes bolivianos en las elecciones de su país de origen, Barcelona, CIDOB, 2012.

Lafleur, Jean-Marie, Von Arx Emmanuel, « "Basta con Evo !”. El voto boliviano en Nueva York », In Diáspora y voto en el exterior. La participación poítica de los emigrantes bolivianos en las elecciones de su país de origen, Barcelona, CIDOB, 2012, p. 129-150.

Lavaud, Jean-Pierre et Isabelle Daillant, La catégorisation ethnique en Bolivie, Paris, L'Harmattan, 2007.

Ledo Garcia, Carmen, « Resultados generales de la Encuesta Nacional de Migraciones Internacionales y Remesas - 2013, Ciudades de La Paz y El Alto, version preliminar », Cochabamba, CEPLAG, 2013.

Le Gall, Julie, Buenos Aires maraîchère : une Buenos Aires bolivienne? Le complexe maraîcher de la Région métropolitaine à l'épreuve de nouveaux acteurs, Thèse de doctorat de géographie, Université de Paris I, 2011.

Marzadro, Mirko, « Conexiones translocales y formacion de territorios migratorios. El caso de los cochabambinos de Bérgamo ", SSIIM Paper series, Unesco Chair Universita IUAV di Venezia, n², 2009.

Parella Rubio, Sonia, Mujer, inmigrante y trabajadora: la triple discriminación, Barcelona, Anthropos, 2003.

Passel, Jeffrey et Vera Cohn, « Population Decline of Unauthorized Immigrants Stalls, May Have Reversed », Pew Research Center, 2013.

Poupeau, Franck, Carnets boliviens (1999-2007). Un goût de poussière, Paris, Aux lieux d'être, 2008.

Price, Marie, « Andean South Americans and Cultural Networks », In Contemporary Ethnic Geographies in America, Rowman and Littlefield, 2006, p. 191-210.

Price, Marie et Elizabeth Chacko, « The mixed embeddedness of ethnic entrepreneurs in a new immigrant gateway », Journal of Immigrant and Refugee Studies, n, 2009, p. 1-19.

Price, Marie, Ivan Cheung, Samantha Friedman et al., " The World Settles in : Washington, DC, as an Immigrant Gateway », Urban Geography, vol. 26, n¹, 2005, p. 61-83.

Price, Marie et Courtney Whitworth, «Soccer and Latino Cultural Space : The Fútbol Leagues of Washington, D.C. », In Hispanic Spaces: Latino Places, Austin, University of Texas Press, 2004, p. $167-186$.

Sassone, Susana, Geografías de la Exclusión. La Inmigración Limítrofe Indocumentada en la Argentina. Del Sistema-Mundo al Lugar, Tesis doctoral en Geoografía, Mendoza, Universidad Nacional de Cuyo, 2002. 
Strunk, Christopher, Circulating citizenship practices : Bolivian routes of migration, hometown associations and development, $\mathrm{PhD}$ in geography, University of Minnesota, 2012.

Strunk, Christopher, « Practicing Citizenship : Bolivian Migrant Identities and Spaces of Belonging in Washington DC », Journal of Intercultural Studies, vol. 36, n5, 2015, p. 620-639.

Taylor, Paul, Marc-Hugo Lopez, Jessica Hamar Martinez et al., « When Labels Don't Fit : Hispanics and Their Views of Identity », Washington DC, Pew hispanic center, 2012.

Vertovec, Steven, « Super-diversity and its implications », Ethnic \& Racial Studies, vol. 30, n6, 2007, p. 1024-1054.

Webner, Pnina, « Global Pathways. Working class cosmopolitans and the creation of transnational ethnic worlds », Social Anthropology, vol. 7, n¹, 1999, p. 17-35.

Yarnall, Kaitlin et Marie Price, « Migration, Development and a New Rurality in the Valle Alto, Bolivia », Journal of Latin American Geography, vol. 9, n¹, 2010, p. 107-124.

\section{NOTES}

1. La loi 94-311 du Congrès états-unien de 1976 oblige la collecte de données concernant les résidents "d'origine mexicaine, cubaine, portoricaine, centraméricaine, sud-américaine et d'autres pays de langue espagnole, indépendamment de leur race", officialisant la création des Hispanics. Une directive de 1997 établit la synonymie du terme d'Hispanic et celui de Latino, que j'utiliserai indifféremment. Depuis le recensement de 2000, leur dénombrement repose sur l'auto-identification de l'origine.

2. 50,3 millions en 2010 , soit $16,4 \%$; 53 millions, soit $17,1 \%$ en 2013 de la population $(12,3 \%$ d'Afro-Américains, 4,7 \% d'Asiatiques, 69,1 \% de Blancs) (données US Census).

3. Sur les près de 12 millions de migrants en situation irrégulière aux États-Unis en 2012 (30\% des individus nés à l'étranger), $80 \%$ sont Latino-Américains, $58 \%$ mexicains (Baby-Collin V., 2014 ; Passel J., Cohn, V., 2013).

4. Les transferts de fonds des migrants oscillent entre 6 et $8 \%$ du PNB : c'est plus que dans la majorité des pays d'émigration de la région (Mexique, Équateur, Pérou), bien moins toutefois que dans les petits pays très dépendants de la migration, comme le Salvador (plus de $16 \%$ du PNB).

5. $89 \%$ des migrants boliviens aux États-Unis sont issus d'origine urbaine, contre $68 \%$ seulement des migrants boliviens toutes destinations confondues, et seulement $56 \%$ de ceux qui se dirigent vers l'Argentine En Bolivie, le taux d'urbanisation en 2012 est de 67 \% (recensement 2012).

6. Les recensements américains permettent de différencier les individus nés à l'étranger de ceux qui se reconnaissent d'une certaine origine, bien qu'étant nés sur le territoire américain, selon les principes de l'auto-détermination des origines (cf. note 1).

7. Les districts qui subdivisent les comtés ne sont représentés que dans les comtés de Prince Williams, Fairfax et Loudoun, où la présence des étrangers et des Latino-américains est la plus importante. Les données sont présentées à l'échelle du comté pour les autres juridictions.

8. Érigée en 1998 au rang de "Patronne de l'intégration nationale", la Vierge d'Urkupiña donne lieu dans la région de Cochabamba à une célébration mariale qui se déploie sur plusieurs jours, chaque 15 août, et attire des dizaines de milliers de fidèles et de badauds. Evo Morales a demandé son inscription sur la liste du patrimoine mondial de l'Humanité de l'UNESCO en 2009.

9. Il est fréquent que les migrants importent en migration des statuettes représentant leur Vierge de prédilection, et de lui dédier un culte dans un cadre privé.

10. Bien que signifiant "de Santa Cruz, le terme englobe les populations de l'Oriente, aussi appelées Cambas. 
11. La Bolivie est l'un des pays latino-américains où la population indienne est la plus nombreuse ; selon le critère de l'auto-identification qui préside aux recensements, elle varie de $41 \%$ (2012) à 62\% (2001) -cf. Lavaud J-P., Daillant I., 2007.

12. Victor Hugo Cárdenas fut le premier vice-président bolivien d'origine aymara (1993-1997).

13. En Espagne, dans le quartier d'Usera, appelé la petite Bolivie de Madrid, ou plus encore en Argentine, dans le quartier de Liniers, à Buenos Aires, ont émergé de véritables centralités ethniques, affichant des scénographies commerciales boliviennes, érigeant ces espaces en lieux ressources.

\section{RÉSUMÉS}

Les États-Unis sont un pôle secondaire dans la migration internationale bolivienne, caractérisée par sa multipolarité. Peu abordée dans les travaux scientifiques consacrés à la présence sudaméricaine en Amérique du nord, cette migration est souvent invisibilisée. Quelles en sont les caractéristiques au regard de la diversité du champ migratoire bolivien, d'une part, et de la présence latino-américaine aux États-Unis, d'autre part ? Comment la faible visibilité du groupe et sa relative hétérogénéité sociale affectent-elles les processus de reconstruction identitaire à l'œuvre dans les espaces de la migration? Cet article met en évidence la diversité des profils de migrants boliviens, statistiquement plutôt qualifiés, et les spécificités de leur présence très concentrée dans la région métropolitaine de Washington, DC. Il analyse comment, entre invisibilité fondue dans une latinité dominante, revendication d'une certaine "bolivianité", et tensions identitaires à la fois sociales et régionales, se redéfinissent les processus d'identification en migration, dans des espaces de vie qui témoignent de l'élaboration progressive de cosmopolitismes populaires. Il s'appuie à la fois sur une analyse statistique et sur une enquête de terrain menée dans la mégalopole atlantique et en Bolivie auprès de migrants aux parcours migratoires et aux modalités d'incorporation dans les métropoles différenciés.

The United States is a secondary destination within the multipolar Bolivian migratory field. Hardly ever mentioned in the scientific production related to the South American presence in North America, this migration is mostly invisible. What are its characteristics, compared with the diversity of the Bolivian migratory field, on one hand, and with the Latin American presence in the United States, on the other ? What are the impacts of its invisibility, as well as its social heterogeneity, on the processes of identity-making that migration produces? This article reveals the diversity of Bolivian profiles, statistically relatively skilled, and the specificities of their concentrated presence in the Washington, D.C. metropolitan area. It analyzes how processes of identity-making are redefined through international migration, between an invisibility diluted within a dominant latinity, the claims to a form of bolivianity, and regional as well as social identifications in tension, in spaces where popular cosmopolitanisms are slowly getting built. It draws from a statistical analysis as well as a qualitative fieldwork carried out in the Atlantic metropolis with Bolivian migrants with diverse trajectories and ways of incorporation in the United States.

Los Estados Unidos forman un polo secundario dentro de la migración internacional boliviana, caracterizada por su multipolaridad. Pocos estudios tratan de esta presencia migrante en 
America del Norte, contribuyendo a invisibilizarla. ¿ Cuáles son las características de esta migración, dentro de la diversidad del campo migratorio boliviano, por un lado, y dentro de la amplia presencia latino-americana en Estados Unidos, por otra parte ? ¿ Cuáles son las consecuencias de la poca visibilidad del grupo asi como de su heterogeneidad social sobre los procesos de reconstrucción identitaria que se dan a ver en la migración ? Este articulo pone en evidencia la diversidad de los perfiles de los migrantes bolivianos, relativamente calificados, y las especificidades de su presencia muy concentrada en la región metropolitana de Washington, D.C. Analiza cómo se redefinen los procesos de identificación a través de la migración, entre una invisibilidad diluida en una latinidad dominante, y tensiones identitarias tanto sociales como relacionadas a las regiones de origen, en espacios de vida donde se elaboran poco a poco formas de cosmopolitismos populares. El texto se apoya tanto sobre un análisis estadístico como sobre una encuesta llevada a cabo en la metrópolis atlántica con migrantes cuyas trayectorias migratorias y modalidades de inserción en el destino son variables.

\section{INDEX}

Palabras claves : campo migratorio, procesos de identificación, Latinos, bolivianos, Estados Unidos

Mots-clés : champ migratoire, processus d'identification, Latinos, Boliviens, États-Unis

Keywords : migratory field, identity-making, Latinos, Bolivians, United States

\section{AUTEUR}

\section{VIRGINIE BABY-COLLIN}

Virginie Baby-Collin est professeure de géographie à l'université d'Aix-Marseille, membre du laboratoire Telemme (UMR7303) et membre de l'Institut Universitaire de France (depuis 2015). Elle coordonne, à la Maison méditerranéenne des sciences de l'homme (MMSH) d'Aix en Provence le réseau thématique de recherches interdisciplinaires sur les migrations, avec Sylvie Mazzella. Elle mène depuis une quinzaine d'années des recherches sur les migrants latinoaméricains dans les métropoles d'Amérique latine, des États-Unis et d'Europe méridionale, envisageant leurs modes d'ancrage, de mobilité et de circulation transnationale. Virginie.babycollin@univ-amu.fr 